\title{
RELIABILITY ANALYSIS OF NONCOHERENT SYSTEMS BY LOGICAL DIFFERENTIAL CALCULUS AND BINARY DECISION DIAGRAMS
}

Reliability is the important characteristic of systems. Most of the systems consist of more than one component. Moreover, modern systems are very complex and they include a lot of components. Therefore, effective methods have to be developed for analysis of these systems. The techniques that have been already considered for this purpose are the Binary Decision Diagrams and the Logical Differential Calculus. However, these methods have been considered only for the coherent systems. In this paper, their use in the reliability analysis of the noncoherent systems is considered.

Keywords: Noncoherent system, binary decision diagram, direct partial logic derivative, importance analysis, strucutral importance measure.

\section{Introduction}

Reliability is one of the basic characteristics of many real systems. During the last decades, there has raised the necessity of reliability analysis of very complex systems [1]. These systems contain a huge amount of components and so there exist some difficulties in analyzing of these systems. One of possible ways to decide this problem is to use special methods and algorithms for processing of large dimension data. Authors of the paper [2] considered for reliability analysis of systems such approach that is named as the Binary Decision Diagram (BDD). The $\mathrm{BDD}$ has been developed for the effective representation of the Boolean functions [3], but they can be also used for the structure function [4], which is used in the reliability analysis to express the correlation between the states of the system components and the state of the whole system.

The suitability of using the BDD for the representation of the structure function results from the fact that the definition of the structure function is formally equivalent with the definition of the Boolean function [5]. This fact allows using the tools for the analyzing of the Boolean functions. The Logical Differential Calculus is one of the techniques that can be also used in the special part of the reliability analysis, which is known as the Importance Analysis [4]. The principal goal of this part of the reliability analysis is to identify the components of the system that are more critical for the proper functioning of the analyzed system [6]. For this purpose, there exist some measures that are known as Importance Measures.

The most attention in the reliability is addressed to the coherent systems, which are characterized by the fact that the failure of the system can be caused only by the failure of the system component, i.e. not by the repair of the component. However, there exist some real systems that do not meet this assumption [7]. These systems are known as the noncoherent systems. Some techniques for the evaluation of the reliability and for the Importance Analysis of these systems have been considered in papers [ $8-11]$. In this paper, the new techniques for the analysis of noncoherent systems using the BDD and the Logical Differential Calculus are suggested.

This paper has the following structure. The section II contains the concept of the system structure function and noncoherent systems, the usefulness of the Logical Differential Calculus in the reliability analysis and the use of the BDD for the representation of the structure function. In the section III, the evaluation of the system reliability of the noncoherent system is considered using the BDD. Finally, the ideas of the Importance Analysis of the noncoherent systems are presented in the section IV. Using the Logical Differential Calculus, the universal definition of the Structural Importance Measure for the noncoherent system and

\footnotetext{
* Jozef Kostolny, Miroslav Kvassay, Stefan Kovalik

Department of Informatics, Faculty of Management Science and Informatics, University of Zilina, Slovakia

E-mail: jozef.kostolny@fri.uniza.sk
} 
the algorithm for its calculation from the BDD of the structure function is developed in this section too.

\section{Mathematical Background}

\section{A.The Structure Function}

Consider the system of $n$ components. Every component can be in one of two states: failed (it is 0 ) and functional (it is 1 ). The state of the whole system depends on the states of its components. Define the state of the $i$-th system component as $x_{i}$, for $i=1, \ldots, n$. Then, the system state can be described mathematically by the structure function [5]:

$$
\phi\left(x_{1}, x_{2}, \ldots, x_{n}\right)=\phi(\boldsymbol{x}):\{0,1\}^{n} \rightarrow\{0,1\},
$$

where $\boldsymbol{x}=\left(x_{1}, \ldots, x_{n}\right)$ denotes the vector of states of the system components. The structure function describes the topological dependencies between individual components and therefore it can be used only for the system reliability analysis from the topological point of view. The reliabilities of individual components must be known to analyze the real (not only topological) reliability of the whole system. Because of that, every component is characterized by probabilities of functional and failed state:

$$
\begin{aligned}
& p_{i}=\operatorname{Pr}\left\{x_{i}=1\right\}, q_{i}=\operatorname{Pr}\left\{x_{i}=0\right\}, p_{i}+q_{1}=1, \\
& i=1,2, \ldots, n .
\end{aligned}
$$

The probability $p_{i}$ represents the reliability of component $i$, while the probability $q_{i}$ represents its unreliability.

\section{B. Coherent and Noncoherent Systems}

According to the properties of the structure function (1), there exist two different types of systems: coherent and noncoherent systems. The coherent system has the following properties [5]:

(i) every component is relevant; i.e. for every component there exist at least one situation, in which the change of the component state results in the change of the system state:

$$
\forall i \in\{1,2, \ldots, n\} \exists\left(1_{i}, \boldsymbol{x}\right): \boldsymbol{\phi}\left(1_{i}, \boldsymbol{x}\right) \neq \boldsymbol{\phi}\left(0_{i}, \boldsymbol{x}\right),
$$

where $(a, \boldsymbol{x})=\left(x_{1}, x_{2}, \ldots, a_{i} \ldots, x_{n}\right)$, for $a \in\{0,1\}$,

(ii) the structure function is non-decreasing; i.e. the failure of any functional component must not cause the repair of the failed system and the repair of any failed component must not result into the repair of the system:

$$
\forall i \in\{1,2, \ldots, n\} \forall\left(1_{i}, \boldsymbol{x}\right): \boldsymbol{\phi}\left(1_{i}, \boldsymbol{x}\right) \neq \phi\left(0_{i}, \boldsymbol{x}\right) .
$$

The noncoherent system does not meet at least one of the assumptions (i) and (ii). Specially, if only assumption (i) is not satisfied, then the system is identified as a monotone or semicoherent system [12].

Most of the systems that are studied in reliability engineering are coherent. However, there exist some real systems that do not satisfy the property (i) or (ii). The typical systems, which are noncoherent, because they do not meet the assumption (ii), are $k$-to-l-out-of- $n$ systems, which real examples in multiprocessor and transportation systems are given in paper [7]. These systems are functional if and only if at least $k$ but not more than $l$ components are working. Other examples of the noncoherent systems have been presented in papers [8] and [10], in which the reliability of liquid-level control-system and gas-detection system has been analyzed.

\section{The Logical Differential Calculus in the Reliability Analysis}

The formal definition of the structure function (1) is identical with the definition of the Boolean function. Therefore, the tools of the Boolean logic and Boolean algebra can be used for the reliability analysis, based on the structure function. One of such tools is the Logical Differential Calculus [13], which allows the investigation of the change of the value of the Boolean function caused by the change of the value of the Boolean variable. In the reliability engineering, the structure function and the system components are used instead of the Boolean function and the Boolean variables. Therefore, the Logical Differential Calculus can be used for the analysis of the influence of the change of the system component state on the system state. The application of this technique for the coherent systems is considered in paper [5].

The Direct Partial Logic Derivative (DPLD) is the tool of the Logical Differential Calculus, which can be used in the reliability analysis [5]. The DPLD $\partial \phi(j \rightarrow \bar{j}) / \partial x_{i}(a \rightarrow \bar{a})$ of a Boolean function $\partial \phi(x)$ of $n$ variables with respect to variable $x_{i}$ reflects the fact of changing the function from $j$ to $\bar{j}$ when the value of variable $x_{i}$ changes from $a$ to $\bar{a}$ [5]:

$$
\begin{aligned}
& \partial \phi(j \rightarrow \bar{j}) / \partial x_{i}(a \rightarrow \bar{a})= \\
& \left\{\phi\left(a_{i}, \boldsymbol{x}\right) \sim j\right\} \wedge\left\{\phi\left(\bar{a}_{i}, \boldsymbol{x}\right) \sim \bar{j}\right\}
\end{aligned}
$$

where $a, j \in\{0,1\}$ and $\sim$ is the symbol of the equivalence operation.

The definition (3) implies that there exist 4 different DPLDs for one variable, which have the following properties [5]:

$$
\begin{aligned}
& \partial \phi(1 \rightarrow 0) / \partial x_{i}(1 \rightarrow 0)=\partial \phi(0 \rightarrow 1) / \partial x_{i}(0 \rightarrow 1),(4) \\
& \partial \phi(1 \rightarrow 0) / \partial x_{i}(1 \rightarrow 0)=\partial \phi(0 \rightarrow 1) / \partial x_{i}(1 \rightarrow 0) .(5)
\end{aligned}
$$

In the reliability analysis, the DPLDs $\partial \phi(1 \rightarrow 0) / \partial x_{i}$ $(1 \rightarrow 0)$ and $\partial \phi(0 \rightarrow 1) / \partial x_{i}(1 \rightarrow 0)$ model the influence of the component's failure on the system state, while the DPLDs $\partial \phi(0 \rightarrow 1) / \partial x_{i}(0 \rightarrow 1)$ and $\partial \phi(1 \rightarrow 0) / \partial x_{i}(0 \rightarrow 1)$ model the consequence of the component's repair. Therefore, the DPLDs 
permit to determine boundary states, i.e. situations, in which the failure or repair of the component results in the change of the system state.

The property (ii) of the coherent system implies that the coherent system meets the following assumption [5]:

$$
\begin{aligned}
& \partial \phi(1 \rightarrow 0) / \partial x_{i}(1 \rightarrow 0)=0 \\
& \text { and } \partial \phi(0 \rightarrow 1) / \partial x_{i}(0 \rightarrow 1)=0 .
\end{aligned}
$$

The consequence of this assumption is that only two DPLDs have to be considered in the case of the analysis of the coherent system. However, the noncoherent systems do not satisfy this assumption and, therefore, all DPLDs have to be analyzed.

\section{The Binary Decision Diagram for the Representation of the Structure Function}

The structure function can be represented in several ways, for example, algebraically (the expression in the terms of the Boolean algebra), tabular (the decision table) and graphically. The BDD is one of graphic forms that are very common [2]. The BDD allows implementation of new algorithms for reliability quantification that are efficient in terms of computational time and accuracy [2].

The construction of the BDD is based on a disjoint decomposition of the Boolean function, which is known as the Shannon expansion [3]. In the terms of the structure function (1), this expansion can be defined as:

$$
\phi(\boldsymbol{x})=x_{i} \phi\left(1_{i}, \boldsymbol{x}\right) \vee \bar{x}_{i} \phi\left(0_{i}, \boldsymbol{x}\right) \text {. }
$$

The BDD is a directed acyclic graph for representation of the Boolean function. It has two sink nodes, labeled by 0 and 1 , which represent two different values (false and true) of the Boolean function. Each non-sink node is labeled with some variable of the Boolean function and has two outgoing edges, labeled by 0 and 1 , which represent the possible values (false and true) of the variable. The BDD has just one source node, which has the same properties as other non-sink nodes. All paths from the source node to the 0 -sink node represent all variables assignments, for which the Boolean function has false value and all paths from the source node to the 1-sink node represent all situations, for which the function is true [3].

In the terms of the reliability analysis, the sink nodes of the BDD present the possible states of the system (failure and functioning). The non-sink node represents a system component. The 0-edge, which leaves the non-sink node, agrees with the fail of the component, while the 1-edge represents the operation state of the component [2]. A path from the source node to the 0 -sink node coincide with one or more situations, in which the system is failed, and a path to the 1-sink represent one or more cases, in which the system is operational.

For example, consider the liquid-level control-system (Fig. 1), which is studied in papers [8] and [10]. This system consists of three relevant components: the pump, the level controller and the level sensor. Also, there are two components, which are irrelevant from the point of view of the reliability analysis: the vessel and the control valve, which never fails. This system is defined in paper [8]: "The pump feeds liquid to the vessel. If the liquid level in the vessel exceeds a specified level, the level sensor sends to the level controller the signal "liquid level in the vessel is high." Upon receiving the signal, the level controller sends a command to the control valve to increase the output flow. The system failure is defined as the situation, when the liquid level in the vessel is higher than specified. The following assumptions are taken:

1. The pump feeds either normal $\left(x_{1}=1\right)$, or too much liquid $\left(x_{1}=0\right)$.

2. The level sensor is functional $\left(x_{3}=1\right)$, or it generates a spurious signal indicating that "liquid level in the vessel is low" even if the liquid level is actually not low $\left(x_{3}=0\right)$.

3. The level controller either fails to respond to any signal from the level sensor (no action is taken by the control valve) $\left(x_{2}=0\right)$, or responds correctly to the signal from the level sensor (the correct control command is sent to the control valve) $\left(x_{2}=1\right)$."

The structure function of this system is defined as follows:

$\boldsymbol{\phi}(\boldsymbol{x})=\left(x_{1} \wedge x_{2}\right) \vee\left(x_{2} \wedge x_{3}\right) \vee\left(x_{1} \wedge \bar{x}_{3}\right)$

The values of the unreliability of the system components are in Table 1 and the BDD of this system is presented in Fig. 1.

The unreliability of the system components

Table 1

\begin{tabular}{|c|c|}
\hline Component & $q_{i}$ \\
\hline$x_{1}$ & $9.90099 \times 10^{-3}$ \\
\hline$x_{2}$ & $7.40740 \times 10^{-2}$ \\
\hline$x_{3}$ & $1.52534 \times 10^{-1}$ \\
\hline
\end{tabular}

Liquid-level control-system [8] The BDD of Liquid-level control-system
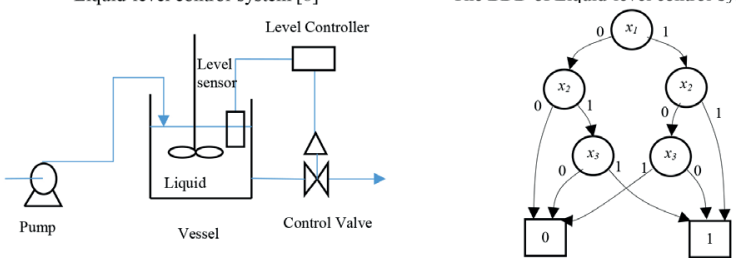

Fig. 1 Liquid-level control-system and its BDD

\section{The System Reliability}

The system reliability is defined with respect to the structure function (1) as the probability, that system is functional [5]:

$$
R=\operatorname{Pr}\{\phi(\boldsymbol{x})=1\}
$$


According to this methodology, the system unreliability is defined as follows [5]:

$$
F=\operatorname{Pr}\{\phi(\boldsymbol{x})=0\}=1-R .
$$

The definitions of the system reliability (7) and the system unreliability (8) can be used in the case of the coherent system as well as in the case of the noncoherent system, because they relate only with the system states regardless of the properties of the structure function.

Now, consider the system, which structural function is defined using the BDD. According to the interpretation of the BDD in the reliability engineering, which is described in the section 2.D, the system reliability (7) and the system unreliability (8) can be defined by the BDDs, which are interpreted as subdiagrams of the BDD of the structure function.

For example, consider the BDD of the liquid-level controlsystem from Fig. 1. The system unreliability can be studied and computed using the first BDD in Fig. 2, while the system reliability can be calculated using the second BDD in Fig. 2.
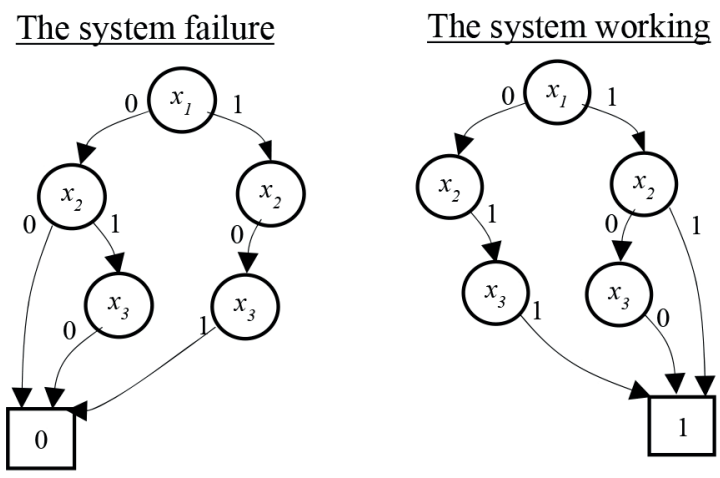

Fig. 2 The failure and working of liquid-level control-system

The reliability of the studied system can be calculated using the second BDD from Fig. 2 as:

$R=q_{1} p_{2} p_{3}+p_{1} q_{2} q_{3}+p_{1} p_{2}=9.925888 \times 10^{-1}$.

The unreliability of the system can be calculated using the first BDD from Fig. 2 as:

$F=q_{1} q_{2}+q_{1} p_{2} q_{3}+p_{1} q_{2} p_{3}=7.411204 \times 10^{-3}$,

or using the definition (8) as:

$F=1-R=7.411204 \times 10^{-3}$.

\section{Importance Analysis}

The system reliability (7) and unreliability (8) are the global characteristics of the analyzed system. However, they have no information value about the importance of individual components for the system. There exist other measures that are used for this purpose. The part of the reliability analysis that studies the influence of individual components on the reliability of the system is known as the Importance Analysis [6]. The Importance Analysis uses some special measures to identify those components, which have the most influence on the function of the system. These measures are known as the Importance Measures (IM).

Most of the IMs have been developed for the analysis of coherent systems. One of possible approaches for calculation of the IMs for the noncoherent system has been considered in paper [9]. Another development of methods for the Importance Analysis of noncoherent systems has been proposed in works [10] and [11], where authors suggested new approach for computation of some IMs for noncoherent systems that included three steps:

1. quantify the influence of the component's failure on the system reliability,

2. quantify the influence of the component's repair on the system reliability,

3. compute the overall influence of the component on the system reliability as the sum of values that have been obtained in steps 1 and 2.

However, some techniques that are considered in papers [10] and [11] cannot be used when the structure function of the noncoherent systems is defined by the BDD. Therefore, another approach for the computation of the IMs is considered in the next part.

\section{A. The Structural Importance Measure for the Non- coherent System}

The Structural Importance Measure (SIM) is one of the simplest measures of the Importance Analysis. It focuses only on the topological aspects of the system. According to [14], this measure determines the proportion of working states of the system in which the working of the component makes the difference between the system failure and its working:

$$
\operatorname{SIM}_{i}=\frac{\rho_{i}}{2^{n-1}}
$$

where $\rho_{i}$ is the number of situations when the change of the component's state results in the failure of the system. In the case of the coherent system, this number can be obtained as the number of nonzero elements of the DPLD $\partial \phi(1 \rightarrow 0) / \partial x_{i}$ $(1 \rightarrow 0)$ [5]. However, in the case of the noncoherent system, also the repair of the system component can cause the failure of the system. Therefore, the number of nonzero elements of the DPLD $\partial \phi(1 \rightarrow 0) / \partial x_{i}(0 \rightarrow 1)$ has to be also considered. So, the SIM for the noncoherent system can be computed as follows:

$$
\begin{aligned}
& \operatorname{SIM}_{i}=\frac{\rho\left(\partial \phi(1 \rightarrow 0) / \partial x_{i}(1 \rightarrow 0)\right)}{2^{n-1}}+ \\
& \frac{\rho\left(\partial \phi(1 \rightarrow 0) / \partial x_{i}(1 \rightarrow 0)\right)}{2^{n-1}}
\end{aligned},
$$


where $\rho($.$) is the function that returns the number of nonzero$ elements of its argument.

The first term in (10) quantifies the consequence of the component's failure on the system functioning, while the second one quantifies the result of the repair of the component on the system functioning. Equation (10) fully agrees with the approach, proposed in paper [10], and moreover it is not fixed to the form, in which the structure function is expressed.

\section{B. Calculation of the Direct Partial Logic Derivatives from the Binary Decision Diagram}

Consider that the structure function of the system has the form of the BDD. To compute the SIM (10), the nonzero elements of the DPLDs $\partial \phi(1 \rightarrow 0) / \partial x_{i}(1 \rightarrow 0)$ and $\partial \phi(1 \rightarrow 0) / \partial x_{i}(0 \rightarrow 1)$ have to be calculated. Some algorithms for this task have been proposed in paper [15]. The algorithms locate two sets of paths in the BDD which satisfy the conditions ( 3 ) for the calculation of the DPLD. In the case of the computation of the DPLD $\partial \phi(j \rightarrow \bar{j}) / \partial x_{i}(a \rightarrow \bar{a})$, the first set consists of the paths that satisfy the condition $\phi\left(\bar{a}_{i}, x\right)=\bar{j}$, i.e. the paths from the source node to the $\bar{j}$-sink node which go through only the $\bar{a}$-edge of the non-sink nodes presented the $i$-th component. The second set includes paths for the condition $\phi\left(a_{i}, \boldsymbol{x}\right)=j$. The comparison of these sets permits to determine paths in the BDD that conform to non-zero values of the DPLD $\partial \phi(j \rightarrow \bar{j}) / \partial x_{i}(a \rightarrow \bar{a})$.

There have been considered some modifications of these algorithms in the paper [4]. These modifications have allowed obtaining two new algorithms. Both new algorithms have identical basic principle. They find the paths from the source node to the sink node that satisfy the conditions (3) for the calculation of the DPLDs. These paths are stored in the special structure that is named as the "Tree of paths" [4].

The first algorithm for calculation of the DPLD $\partial \phi(j \rightarrow \bar{j}) /$ $\partial x_{i}(a \rightarrow \bar{a})$ includes three steps:

1. calculate the "Tree of paths" for the condition $\phi(\bar{a}, \boldsymbol{x})=\bar{j}$,

2. calculate the "Tree of paths" for the condition $\phi\left(a_{i}, \boldsymbol{x}\right)=j$,

3. calculate the intersection of the "Trees of paths" from steps 1 and 2 .

In the second algorithm only two steps are performed:

4. calculate the "Tree of paths" for the condition $\phi\left(\bar{a}_{i}, x\right)=\bar{j}$,

5. check which paths from the "Tree of paths" exist in the sub-diagram of the BDD that fulfill the condition $\phi\left(a_{i}, \boldsymbol{x}\right)=j$

For example, consider the calculation of the DPLDs from the BDD depicted in Fig. 1 using the first algorithm. In Fig. 3, there is shown its work in the case of the calculation of the DPLDs $\partial \phi(1 \rightarrow 0) / \partial x_{3}(1 \rightarrow 0)$ and $\partial \phi(1 \rightarrow 0) / \partial x_{3}(0 \rightarrow 1)$. The second algorithm is very similar to the first one and its work is closely described in paper [4].

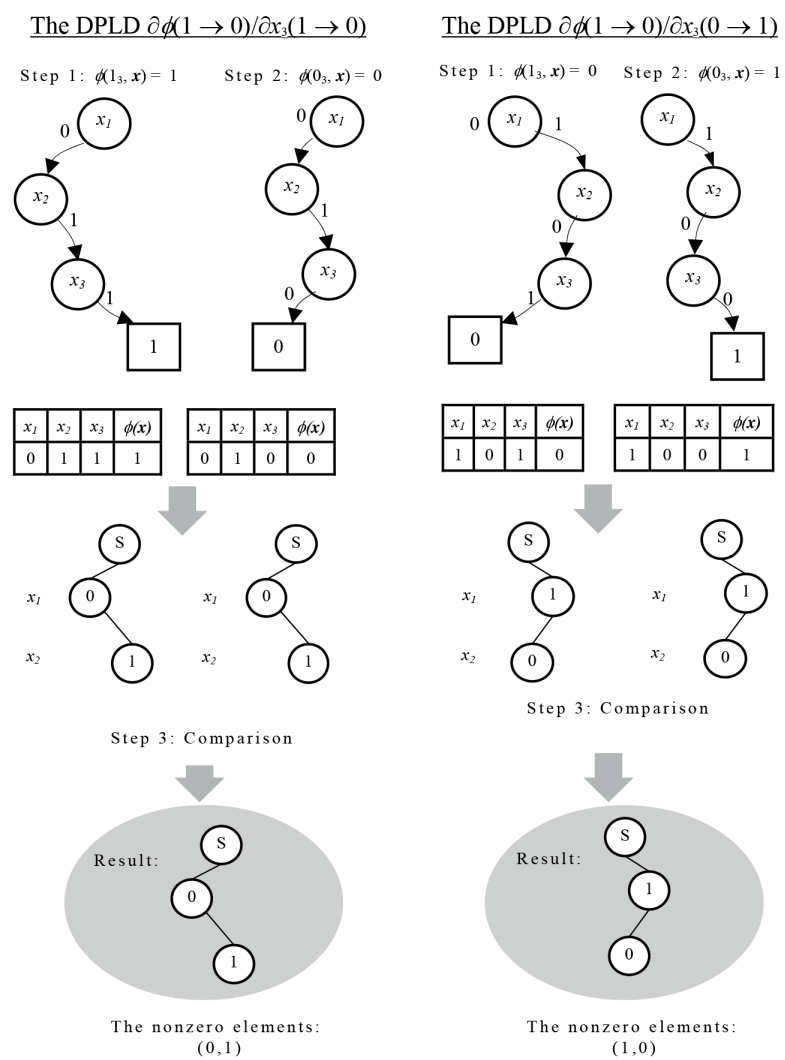

Fig. 3 The first algorithm for the calculation of DPLDS for the third component of liquid-level control-system

\section{The Structural Importance Measures for the Liquid- -Level Control-System}

Consider the system in Fig. 1. The nonzero elements of DPLDs $\partial \phi(1 \rightarrow 0) / \partial x_{i}(1 \rightarrow 0)$ and $\partial \phi(1 \rightarrow 0) / \partial x_{i}(0 \rightarrow 1)$, for $i=1,2,3$, have to be found to calculate the SIMs of its components according to (10). These nonzero elements can be found using some algorithm from the previous section (Table 2). When the nonzero elements of individual DPLDs are known, their count can be determined (Table 2). Finally, the SIMs (10) can be computed (Table 2). It is clear from Table 2 that all components of the system from Fig. 1 have the same value of the SIM and, therefore, there exists no component, which has dominant influence on the system reliability from the topological point of view. 
The nonzero elements of the DPLDs and the SIMs of individual components

Table 2

\begin{tabular}{|c|c|c|c|c|}
\hline Component & DPLD & Nonzero elements of DPLD & Number of nonzero elements of DPLD & SIM \\
\hline \multirow{3}{*}{$x_{1}$} & $\partial \phi(1 \rightarrow 0) / \partial x_{1}(1 \rightarrow 0)$ & $(0,0)(1,0)$ & 2 & 0.5 \\
\cline { 2 - 5 } & $\partial \phi(1 \rightarrow 0) / \partial x_{1}(0 \rightarrow 1)$ & - & 2 & 0 \\
\hline \multirow{3}{*}{$x_{2}$} & $\partial \phi(1 \rightarrow 0) / \partial x_{2}(1 \rightarrow 0)$ & $(0,1)(1,1)$ & 0 & 0.5 \\
\cline { 2 - 6 } & $\partial \phi(1 \rightarrow 0) / \partial x_{2}(0 \rightarrow 1)$ & - & 1 & 0.5 \\
\hline \multirow{2}{*}{$x_{3}$} & $\partial \phi(1 \rightarrow 0) / \partial x_{3}(1 \rightarrow 0)$ & $(0,1)$ & 1 & \\
\cline { 2 - 6 } & $\partial \phi(1 \rightarrow 0) / \partial x_{3}(0 \rightarrow 1)$ & $(1,0)$ & & 0 \\
\end{tabular}

\section{Conclusion}

Some of the modern challenges of the reliability analysis are the analysis of large systems, which contain a lot of interconnected components, and the research of the noncoherent systems which have some properties that have not been considered in the classical approaches of the reliability analysis. The BDD is an efficient tool for the representation of the Boolean functions. The Boolean function can be interpreted as two-valued function. Also the structure function, that defines the structure of the analyzed system, is two valued function. Therefore, the BDD can be used for the effective representation of the structure function. The usefulness of this tool in the reliability analysis of coherent systems has been presented in papers [2] and [4], where the calculations of the system reliability and IMs have been considered.
In this paper the reliability analysis of noncoherent systems is considered. It is shown that the BDD can also be used for the representation of these systems and that the BDD can be used for the evaluation of the reliability and the unreliability of the noncoherent system.

The Importance Analysis of the noncoherent system is considered in the last section of this paper. The definition of the SIM is proposed using the DPLDs. This definition is consistent with the approach which has been suggested in work [10], but moreover, it does not depend on the form of the structure function. Also, the algorithm for the calculation of the SIM for the noncoherent system from the BDD is proposed. The algorithm is based on the procedures for the calculation of the DPLD from the BDD which have been suggested in paper [4].

\section{References}

[1] ZIO, E.: Reliability Engineering: Old Problems and New Challenges, Reliability Engineering \& System Safety, vol. 94, No. 2, pp. 125-141, 2009.

[2] CHANG, Y., AMARI, S.: Computing System Failure Frequencies and Reliability Importance Measures using OBDD, IEEE Transactions on Computers, vol. 53, No. 1, pp. 54-68, 2004.

[3] BRYANT, R. E.: Graph-Based Algorithms for Boolean Function Manipulation, IEEE Transactions on Computers, vol. C-35, No. 8, pp. 677-691, 1986

[4] KOSTOLNY, J., KVASSAY, M., KOVALIK, S.: Analysis of System Reliability by Logical Differential Calculus and Decision Diagrams, Intern. Conference on Digital Technologies (DT), 2013, pp. 170-175.

[5] ZAITSEVA E. N., LEVASHENKO, V. G.: Importance Analysis by Logical Differential Calculus, Automation and Remote Control, vol. 74, No. 2, pp. 171-182, 2013.

[6] KUO, W., ZHU, X.: Importance Measures in Reliability, Risk, and Optimization. Chichester, John Wiley \& Sons, Ltd, 2012.

[7] UPADHYAYA, S. J., PHAM, H.: Analvsis of Noncoherent Systems and an Architecture for the Computation of the System Reliability, IEEE Transactions on Computers, vol. 42, No. 4, pp. 484-493, 1993.

[8] INAGAKI, T., HENLEY, E. J.: Probabilistic Evaluation of Prime Implicants and Top-events for Non-coherent Systems, IEEE Transactions on Reliability, vol. 3, No. 5, pp. 361-367, 1980.

[9] JACKSON, P. S.: On the s-importance of Elements and Prime Implicants of Non-coherent Systems, IEEE Transactions on Reliability, vol. R-32, No. 1, pp. 21-25, 1983. 


\section{COMMNICOIIONS}

[10] ANDREWS, J. D., BEESON, S.: Birnbaum's Measure of Component Importance for Noncoherent Systems, IEEE Transactions on Reliability, vol. 52, No. 2, pp. 213-219, 2003.

[11] BEESON, S. , ANDREWS, J. D.: Importance Measures for Non-coherent-system Analysis, IEEE Transactions on Reliability, vol. 52, No. 3, pp. 301-310, 2003.

[12] KUO, W., ZHU, X.: Relations and Generalizations of Importance Measures in Reliability, IEEE Transactions on Reliability, vol. 61, No. 3, pp. 659-674, 2012.

[13] AKERS, S. B., Jr.: On a Theory of Boolean Functions, J. of the Society for Industrial and Applied Mathematics, vol. 7, No. 4, p. 487, 1959.

[14] ARMSTRONG, M. J.: Reliability-importance and Dual failure-mode Components, IEEE Transactions on Reliability, vol. 46, No. 2, pp. 212-221, 1997

[15] ZAITSEVA, E., LEVASHENKO, V.: Multiple-Valued Logic Mathematical Approaches for Multi-state System Reliability Analysis, J. of Applied Logic, vol. 11, No. 3, pp. 350-362, 2013. 